\title{
Isotopic Constraints on the Origin and Evolution of Martian Volatiles
}

\author{
KUN WANG (王昆) ${ }^{1 *}$ \\ ${ }^{1}$ Department of Earth and Planetary Sciences and McDonnell \\ Center for the Space Sciences, Washington University in \\ St. Louis, MO 63130,USA (wangkun@wustl.edu)
}

Understanding the origin, history, and behavior of water and other volatiles on Mars is crucial to understanding the evolution of Mars, the next frontier for human exploration. To characterize the volatiles of bulk Mars and compare to those of other terrestrial planetary bodies, several elemental and isotopic ratios have been developed over the past few decades [1-6], such as D/H, $(\mathrm{Na}, \mathrm{Ga}, \mathrm{Br}) / \mathrm{Al}, \mathrm{K} /(\mathrm{La}, \mathrm{Th}, \mathrm{U}), \mathrm{Mn} / \mathrm{Na}$, and $\mathrm{Rb} / \mathrm{Sr}$, which can be acquired through either direct analysis on martian meteorites or remote sensing of the martian surface (e.g., Odyssey GRS). According to these indexes, Mars has a higher inventory of volatiles than Earth. Therefore, Mars can be viewed as a volatile-rich (hence water-rich) planet $[3,7]$.

Although this conclusion is consistent with Mars' greater heliodistance and has since become the current paradigm, it is sharply contradictory to the present-day conditions of Mars vs. Earth. One could explain this discrepancy with solar wind stripping mechanism as recently observed by MAVEN. However, how much volatiles have been removed over geological history through this mechanism remains uncertain and so it is unclear whether this alone can justify the difference. Here I will reassess several key geochemical and isotopic constraints for the volatile budgets of Mars and Earth. Then I will introduce $\mathrm{K}$ isotopes, a newly proposed tracer for comparing planetary volatiles [8]. New data suggest that Mars was in fact more depleted in volatiles than Earth (see Fig. 1), which implies much less water was initially accreted to Mars than previously thought.

References: [1] Wasserburg et al. (1964) Science. [2] Morgan \& Anders (1979) GCA. [3] Dreibus \& Wänke (1985) Meteorit. [4] Dreibus \& Wänke (1987) Icarus. [5] Taylor (2013) Chem Erde. [6] Yoshizaki \& McDonough (2020) GCA. [7] Carr (1986) Icarus. [8] Tian et al. (2020) LPSC \#2572.

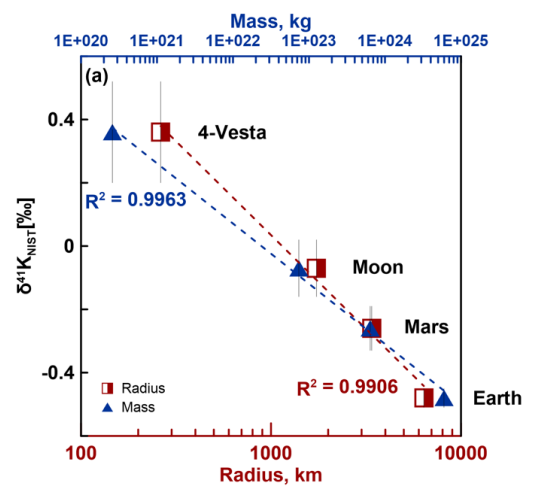

Fig. 1. K isotopic compositions of Earth, Mars, Moon, and Vesta from ref. [8]. 